\title{
TWO APPROXIMATION METHODS OF SPATIAL DERIVATIVES ON UNSTRUCTURED TRIANGULAR MESHES AND THEIR APPLICATION IN COMPUTING TWO DIMENSIONAL FLOWS
}

\author{
Nguyen Duc Lang ${ }^{1}$, Tran Gia $\mathrm{LiCH}^{2}$, and Le DuC ${ }^{3}$ \\ ${ }^{1}$ Falcuty of Natural Science, Thai Nguyen University \\ ${ }^{2}$ Institute of Mathematics \\ ${ }^{3}$ National Center of Hydrological-Meteorological Forecast
}

\begin{abstract}
Two approximation methods (the Green's theorem technique and the directional derivative technique) of spatial derivatives have been proposed for finite differences on unstructured triangular meshes. Both methods have the first order accuracy. A semi-implicit time matching methods beside the third order Adams-Bashforth method are used in integrating the water shallow equations written in both non-conservative and conservative forms. To remove spurious waves, a smooth procedure has been used. The model is tested on rectangular grids triangularized after the 8-neighbours strategy. In the context of the semi-implicit time matching methods, the directional derivative technique is more accurate than Green's theorem technique. The results from the third order Adams-Bashforth scheme are the most accurate, especially for discontinuous problems. In this case, there is a minor difference between two approximation techniques of spatial derivatives.
\end{abstract}

\section{INTRODUCTION}

Models simulating flow in rivers, coastal areas, ... are needed to resolve many natural phenomena in such domains. Because natural phenomena range from small scales to large scales, meshes used in models must vary and depend on problem geometries. That's why unstructured meshes are more appropriate than structured, uniform meshes in modeling flows [7]. The popular methods using unstructured meshes consist of finite volumes and finite elements. A cell, e.g. a triangular, is a base element in such methods. The finite volume method is more preferable than the finite element method because its conservative form of equations implies the conservation of momentum and mass in the results. 'The key concept involves an algorithm specifying the fluxes between two cells.

In finite differences, uniform meshes (usually equispace rectangular grids) are widely used. This may be derived from the approximation technique using the 'Taylor's serie expansion. In this paper we try to approach spatial derivative approximation using other methods. Although the methods are simple, their application is directly consistence with unstructured meshes and easy in implementation.

\section{FORMULATION OF THE NUMERICAL MODEL}

\subsection{Fundamental equations}

The numerical model is based on the two dimensional Saint Venant equations in the non-conservative form [8] 


$$
\begin{gathered}
\frac{\partial u}{\partial t}+u \frac{\partial u}{\partial x}+v \frac{\partial u}{\partial y}+g \frac{\partial(h+z)}{\partial x}-\operatorname{diff}(u)-l v+\frac{\tau_{b x}}{\rho h}-\frac{\tau_{w x}}{\rho h}=0 \\
\frac{\partial v}{\partial t}+u \frac{\partial v}{\partial x}+v \frac{\partial v}{\partial y}+g \frac{\partial(h+z)}{\partial y}-\operatorname{diff}(v)+l u+\frac{\tau_{b y}}{\rho h}-\frac{\tau_{w y}}{\rho h}=0 \\
\frac{\partial h}{\partial t}+\frac{\partial h u}{\partial x}+\frac{\partial h v}{\partial y}=0
\end{gathered}
$$

or in the conservative form (Madsen, 1997)

$$
\begin{gathered}
\frac{\partial p}{\partial t}+\frac{\partial}{\partial x}\left(\frac{p^{2}}{h}\right)+\frac{\partial}{\partial y}\left(\frac{p q}{h}\right)+g h \frac{\partial(h+z)}{\partial x}-\operatorname{diff}(p)-l q+\frac{\tau_{b x}}{\rho}-\frac{\tau_{w x}}{\rho}=0 \\
\frac{\partial q}{\partial t}+\frac{\partial}{\partial x}\left(\frac{p q}{h}\right)+\frac{\partial}{\partial y}\left(\frac{q^{2}}{h}\right)+g h \frac{\partial(h+z)}{\partial y}-\operatorname{diff}(q)+l p+\frac{\tau_{b y}}{\rho}-\frac{\tau_{w y}}{\rho}=0 \\
\frac{\partial h}{\partial t}+\frac{\partial p}{\partial x}+\frac{\partial q}{\partial y}=0
\end{gathered}
$$

where $u, v$ : the depth-averaged current velocity; $p, q$ : the volume flux; $h$ : the instantaneous water depth; $z$ : the bed elevation; $g$ : the gravity acceleration; diff: the diffusion term (the turbulent momentum transfer); $l$ : the Coriolis parameter; $\rho$ : the water density; $\tau_{b x}, \tau_{b y}$ : the bed stress; $\tau_{w x}, \tau_{w y}$ : the wind stress.

The diffusion terms are formulated as the second, fourth or sixth order turbulent momentum transfer scheme. With an appropriate scheme, the diffusion terms will damp spurious waves occurring in the integration and guarantee the stability of numerical schemes.

\subsection{Approximations of spatial derivatives}

Supposed that $f$ is a function we want to calculate its partial spatial derivatives. Suitable approximations of these derivatives are necessary because the model is designed for unstructured meshes instead of rectangular grids. There are two techniques enabling the calculations of spatial derivatives: the Green's theorem technique and the directional derivative technique.

\subsubsection{Green's theorem technique}

Let $M$ be a point that its spatial derivatives have to be approximated and we will numerate the points that link with $M$ in the unstructured triangular mesh in the sequence $1,2, \ldots, n$ (Fig. 1). The area of the polygon made of the edges $12,23, \ldots, n_{1}$ is $S$. Apply the Green's theorem for spatial derivatives of $\mathrm{f}$ with an integration over $S$ and denote the closed contour of the polygon by $C$ we have

$$
\begin{gathered}
\iint_{S} \frac{\partial f}{\partial x} d S=\oint_{C} f \cos (\vec{n}, x) d C=\oint_{C} f d y \\
\iint_{S} \frac{\partial f}{\partial y} d S=-\oint_{C} f \cos (\vec{n}, y) d C=-\oint_{C} f d x
\end{gathered}
$$




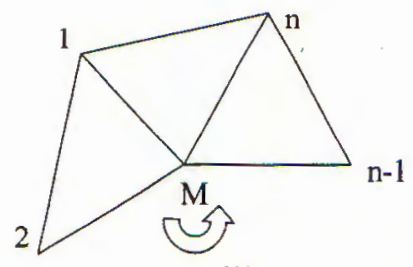

Fig. 1. A sketch of unstructured grid points in formulating spatial derivative approximations after the Green's theorem technique



Fig. 2. A sketch of unstructured grid points in formulating spatial derivative approximations after the directional derivative technique.

Here $n$ is the unit vector normal to the closed contour $C$. Now assuming a piecewise constant approximation to the spatial derivatives inside the polygon $S$, the spatial derivatives at $M$ can be calculated from the following approximations

$$
\begin{aligned}
& \left.\frac{\partial f}{\partial x}\right|_{M}=\frac{1}{S} \oint_{C} f d y+O\left(h_{x}\right) \\
& \left.\frac{\partial f}{\partial y}\right|_{M}=-\frac{1}{S} \oint_{C} f d x+O\left(h_{y}\right)
\end{aligned}
$$

in which $h_{x}, h_{y}$ are the maximum distances from $M$ to the vertices of the polygon $S$ in $x$-axis and $y$-axis respectively.

Two integrations over the closed contour $C$ in the equation $(2.4 \mathrm{a}, \mathrm{b})$ are the sum of the integrations over the edges $12,23, \ldots, n_{1}$ and with a simple linear approximation on each integration, they become

$$
\begin{aligned}
\oint_{C} f d y & =\int_{12} f d y+\int_{23} f d y+\ldots+\int_{n 1} f d y \\
& =\frac{f_{1}+f_{2}}{2}\left(y_{2}-y_{1}\right)+\frac{f_{2}+f_{3}}{2}\left(y_{3}-y_{2}\right)+\cdots+\frac{f_{n}+f_{1}}{2}\left(y_{1}-y_{n}\right) \\
\oint_{C} f d x & =\int_{12} f d x+\int_{23} f d x+\ldots+\int_{n 1} f d x \\
& =\frac{f_{1}+f_{2}}{2}\left(x_{2}-x_{1}\right)+\frac{f_{2}+f_{3}}{2}\left(x_{3}-x_{2}\right)+\ldots+\frac{f_{n}+f_{1}}{2}\left(x_{1}-x_{n}\right)
\end{aligned}
$$

For the area $S$, we can also apply the Green's theorem

$$
S=\iint_{S} d S=\oint_{C} x d y=-\oint_{C} y d x
$$

and it has the same formula as in $(2.5 \mathrm{a}, \mathrm{b})$ where $f$ should be $x$ or $y$.

All the higher order spatial derivatives can be estimated in the same way. To calculate the nth spatial derivatives, we have to compute all $(n-1)^{\text {th }}$ spatial derivatives for all points in the domain then apply the formula (2.5a, b) with $f$ becoming $f^{(n-1)}$. 


\subsubsection{Directional derivative technique}

If $n$ is a vector and the angle between $n$ and the unit vector on the $x$ axis $i$ is $\alpha$, the following formula is always true for the spatial derivative of $f$ in the direction of $n$

$$
f_{\vec{n}}^{\prime}=\frac{\partial f}{\partial x} \cos \alpha+\frac{\partial f}{\partial y} \sin \alpha
$$

Suppose that $O$ is the point where we want to calculate the spatial derivatives. To approximate its spatial derivatives, two spatial derivatives at the point $O$ with respect to two next points $P$ and $Q$ will be considered (Fig. 2). Take $n$ as the two vector $O P$ and $O Q$, then denote their angles with the vector $i$ by

$$
\alpha_{P}=\widehat{O P}, \vec{i} ; \alpha_{Q}=\widehat{O Q}, \vec{i}
$$

Applying (2.7) for the two directions $O P$ and $O Q$ we have

$$
\begin{aligned}
& f_{\overrightarrow{O P}}^{\prime}=\left.\frac{\partial f}{\partial x}\right|_{O} \cos \alpha_{P}+\left.\frac{\partial f}{\partial y}\right|_{O} \sin \alpha_{P}=\frac{f_{P}-f_{O}}{O P}+O(O P) \\
& f_{\overrightarrow{O Q}}^{\prime}=\left.\frac{\partial f}{\partial x}\right|_{O} \cos \alpha_{Q}+\left.\frac{\partial f}{\partial y}\right|_{O} \sin \alpha_{Q}=\frac{f_{Q}-f_{O}}{O Q}+O(O Q)
\end{aligned}
$$

Neglecting the high order terms in $(2.8 \mathrm{a}, \mathrm{b})$, the equations become a linear system. After some simple steps, we retrieve the solution

$$
\begin{aligned}
& \left.\frac{\partial f}{\partial x}\right|_{O}=\frac{1}{2 S}\left[f_{O}\left(y_{Q}-y_{P}\right)+f_{P}\left(y_{O}-y_{Q}\right)+f_{Q}\left(y_{P}-y_{O}\right)\right] \\
& \left.\frac{\partial f}{\partial y}\right|_{O}=-\frac{1}{2 S}\left[f_{O}\left(x_{Q}-x_{P}\right)+f_{P}\left(x_{O}-x_{Q}\right)+f_{Q}\left(x_{P}-x_{O}\right)\right]
\end{aligned}
$$

Now return to the Fig. 1 and apply $(2.9 \mathrm{a}, \mathrm{b})$ for all triangulars $M 12, M 23, \ldots, M n 1$ we get $\mathrm{n}$ estimations for each spatial derivative at the point $M$. The simplest way to calculate a spatial derivative is to average all estimations

$$
\begin{aligned}
& \left.\frac{\partial f}{\partial x}\right|_{O}=\frac{1}{n}\left(\left.\frac{\partial f}{\partial x}\right|_{M 12}+\left.\frac{\partial f}{\partial x}\right|_{M 23}+\ldots+\frac{\partial f}{\partial x}{ }_{M n 1}\right) \\
& \left.\frac{\partial f}{\partial y}\right|_{O}=\frac{1}{n}\left(\left.\frac{\partial f}{\partial y}\right|_{M 12}+\left.\frac{\partial f}{\partial y}\right|_{M 23}+\ldots+\frac{\partial f}{\partial y_{M n 1}}\right)
\end{aligned}
$$

Calculating the higher order spatial derivatives has the same approach like the Green's theorem technique in 2.2.1.

\subsection{Time matching methods}

There are many time matching methods (see for example in Lomax, 1999) and we can choose an appropriate method with spatial derivative approximations in (2.2). The third order Adams-Bashforth scheme is a good candidate because its highly accurate (third order in time) and economical (explicit method) property. This scheme will be used for the equations in the conservative form (2.2). Suppose that $f$ is a function varied in time, then the value of $f$ in the future can be updated from the current value and the time derivatives in the past

$$
f^{n+1}=f^{n}+\frac{1}{12}\left(23{\frac{\partial f^{n}}{\partial t}}^{n}-16 \frac{\partial f}{\partial t}^{n-1}+5 \frac{\partial f}{\partial t}^{n-2}\right) \Delta t
$$


For the non-conservative form, the semi implicit approach is taken in handling the advection terms. These nonlinear terms always request special attentions. Here are three semi implicit integrating method.

Rewriting the equations $(2.1 \mathrm{a}, \mathrm{b}, \mathrm{c})$

$$
\begin{gathered}
\frac{\partial u}{\partial t}+u \frac{\partial u}{\partial x}=F_{u} \\
\frac{\partial v}{\partial t}+v \frac{\partial v}{\partial y}=F_{v} \\
\frac{\partial h}{\partial t}+h\left(\frac{\partial u}{\partial x}+\frac{\partial v}{\partial y}\right)=F_{h}
\end{gathered}
$$

and discretizing all terms in the following form

$$
\begin{gathered}
\frac{u_{i}^{n}-u_{i}^{n-1}}{\Delta t}+\left.u_{i}^{n} \frac{\partial u}{\partial x}\right|_{i} ^{n-1}=F_{u i}^{n-1} \\
\frac{v_{i}^{n}-v_{i}^{n-1}}{\Delta t}+\left.v_{i}^{n} \frac{\partial v}{\partial y}\right|_{i} ^{n-1}=F_{v i}^{n-1} \\
\frac{h_{i}^{n}-h_{i}^{n-1}}{\Delta t}+\left.h_{i}^{n}\left(\frac{\partial u}{\partial x}+\frac{\partial v}{\partial y}\right)\right|_{i} ^{n-1}=F_{h i}^{n-1}
\end{gathered}
$$

As usual in the CFD context, subscript indices denote space indices while superscript indices denote time indices. All spatial derivatives in $(2.13 a, b, c)$ are estimated from the methods in the part 2.2. The explicit solutions for the equations $(2.13 a, b, c)$ can be easily found and are not shown here.

Actually the semi-implicit method are more complex than the above description. For each time step an iterative procedure is done to promote the accuracy of solutions. With an error percentage is 1 , the number of iterative steps is from 2 to 5 .

\subsection{Boundary conditions}

Without the diffusion term, the equations (2.1) can be transformed into the symmetric form which is quasi-linear hyperbolic. The eigenvalues of the flux Jacobian matrix are phase speeds of waves travel in or out the domain. The wave speeds depend on the normal velocity $u_{n}$ and the gravity wave velocity $c$. The number of boundary conditions is the same the number of waves traveling in the domain. So the number boundary conditions are problem-oriented and we need a general frame in implementing boundary conditions. Here are boundary conditions supported in the model

Imposed boundary conditions which may be flow velocity $u, v$, discharge $p, q$ or water $\operatorname{depth} h$.

Solid boundary conditions.

Radiative boundary conditions.

Depending on the number of boundary conditions, the complementary equations have to be specified on the boundary or not. Using the characteristics method, Tran et al. [8] founded these equations when the boundary is parallel to the coordinate axis. Because the model is based on unstructured triangular meshes, these supplementary equations can't be applied directly and we will chose a more simple approach. If a variable is not specified on the boundary, its value is calculated from its difference equation. 


\subsection{Smoothing}

In testing the model with shock waves or supercritical flows, high frequency oscillations occur in the solutions, amplify very fast and overcome all slow waves. To smooth out such waves from solutions, we use a smooth procedure. Smoothing will be carried out at a given time for all points after a given step. For a field like $h$, after each smooth step, its value at a point $M$ (Fig. 1) will be

$$
h_{M}=(1-w) * h_{M}+w * \overline{h_{M}}
$$

where $w$ is the smooth weight ( 0.02 in this model) and the average of $h$ is computed from the surrounding points

$$
\overline{h_{M}}=\frac{1}{n}\left(h_{1}+h_{2}+\ldots+h_{n}\right)
$$

With the smooth formulation (2.14) the conservation of mass may be violated but we found that it is not significant in practice as shown in the following section.

\section{MODEL TESTING}

In the following section some tests are carried out to validate the model performance. To simplify the output handling, all computational points will be chosen from vertices of a rectangular grid. However, all are considered in the context of unstructured triangular meshes. Fig. 3 shows some strategies generating a triangular mesh from points in a rectangular grid. All tests are based on the 8-neighbours strategy.
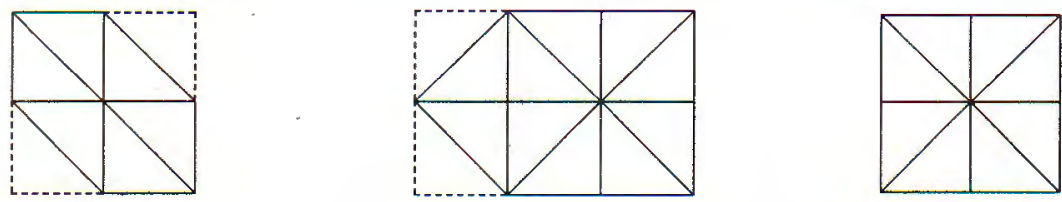

Fig. 3. Three strategies generating unstructured triangular meshes from rectangular grids: 6-neighbours (left), 4-8-neighbours (center), 8-neighbours (right).

Two spatial derivative approximation techniques, the Green's theorem and the directional derivative, will be denoted by $S 1$ and $S 2$ respectively. $T 1$ is a short symbol for three semi-implicit time matching methods represented in 2.3. T2 is for the third order Adams-Bashforth scheme. In all figures, the analytical solution (optional) will be shown by a dash line and the numerical solution a solid line. Dashed lines are also used for bed elevations in some figures. A local context will make the meaning obvious.

\subsection{Dam break over a wet or dry bed}

The problem configuration is shown in Fig. 4 (left). There is a dam between two water layers with the depths are $h_{r}$ and $h_{l}$ respectively. The dam is supposed to vanish instantaneously. This problem enables testing the treatment of the free surface gradient and the wetting - drying handling. Fig. 4 (right) plots the analytical solution in the wet bed case without friction.

In testing we set $h_{l}=6 \mathrm{~m}, h_{r}=2 \mathrm{~m}$. The channel length is $1000 \mathrm{~m}$, the channel width is $4 \mathrm{~m}$ and the distance between two successive points is $2 \mathrm{~m}$. The zero discharge is imposed in left and right boundaries. No friction and diffusion forces are included. The numerical solution compared to the numerical solutions after $30 \mathrm{~s}$ from all spatial 

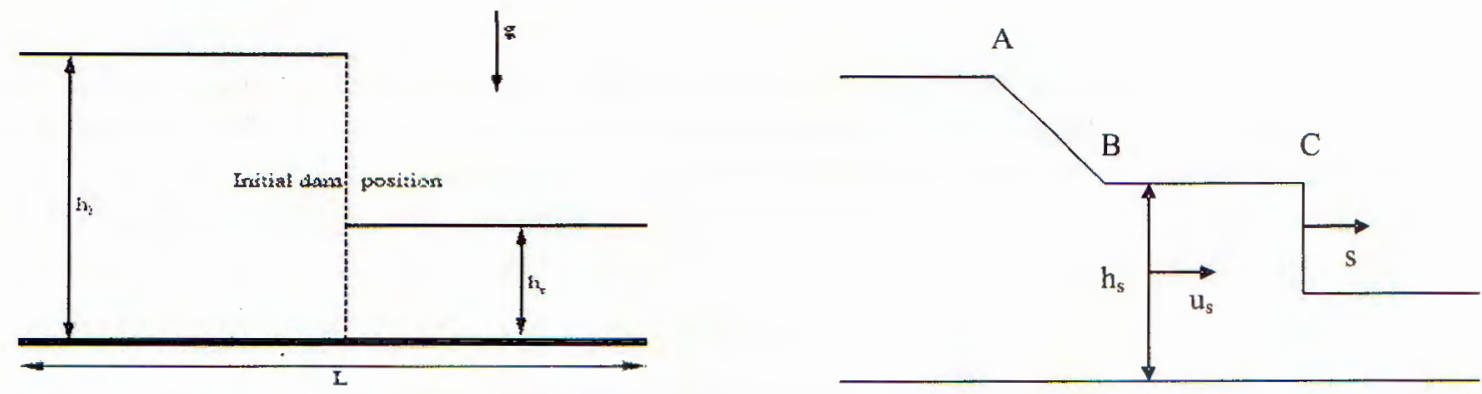

Fig. 4. The geometry of dam breaks over a wet bed (left) and the analytical solution of dam breaks over a wet bed when bottom frictions are ignored (right).
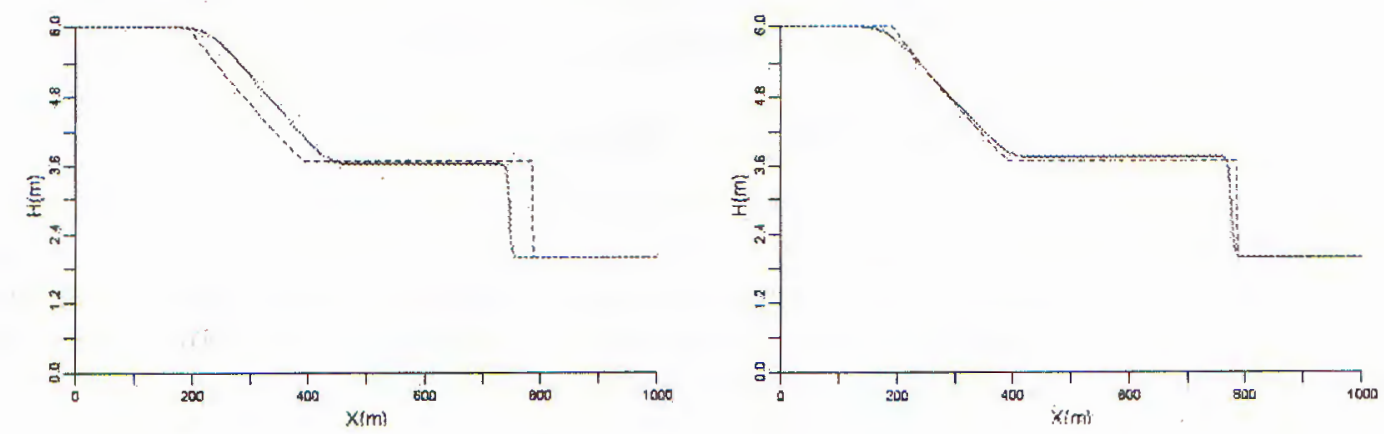

Fig. 5. The numerical solutions compared to the analytical solutions after 30 s for dam break over a wet bed, which result from the semi-implicit approach. The results with the spatial scheme S1 are in the left and S2 in the right.

derivative techniques combined with the semi implicit integration methods and the third order Adams-Bashforth scheme is shown in Figs. 5 and 6. The time step is $0.1 \mathrm{~s}$ and the number of smoothing is 5 for each time step.
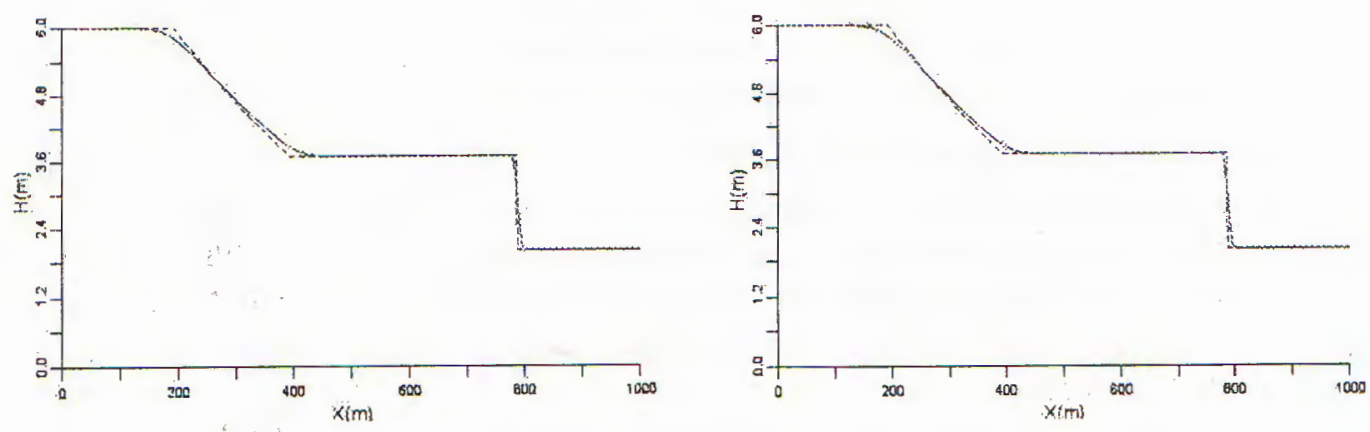

Fig. 6. The numerical solutions compared to the analytical solutions after $30 \mathrm{~s}$ for dam break over a wet bed, which result from the third order Adams-Bashforth approach. The results with the spatial scheme $S 1$ are in the left and $S 2$ in the right. 
Using any semi-implicit method, the numerical solution phase is slower than the numerical analytical phase. However, the numerical solutions from $S 2$ are more accurate than those from $S 1$. But this is not true in case of the third order Adams-Bashforth scheme $(T 2)$ as in the Fig. 6 where the numerical solutions are identical. And the first technique $S 1$ will be combined with the scheme $T 2$ in the next tests.

Comparing the Fig. 6 to Fig. 7, the third order Adams-Bashforth scheme seems to be more attractive than the semi-implicit approach. This is asserted in simulating dam break over dry bed (Fig. 7). All initial and boundary conditions are the same except $h_{r}$ is set to zero. The integration time is $30 \mathrm{~s}$.
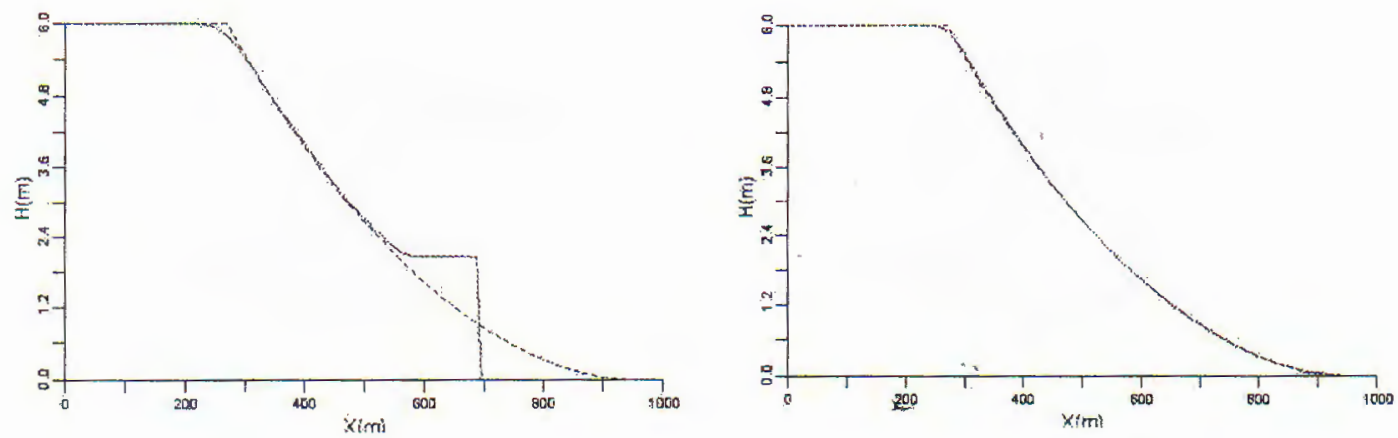

Fig. 7. The numerical solutions compared to the analytical solutions after $30 \mathrm{~s}$ for dam break over a dry bed, which use the spatial scheme $S 1$. The results with the semi-implicit scheme $T 1$ are in the left and the scheme $T 2$ in the right.

While the third order Adams-Bashforth can catch very well the analytical solution, the semi-implicit approach misses the true solution with a numerical shock wave. Fig. 7 also proves the good wetting - drying treatment in the model.

\subsection{Partial dam break}

This problem is a general case in two dimensional space of the above problem. It is proposed by Fennema (1990). All initial conditions remain in the one dimensional case where a dam dividing the domain into two same water layers with different depths. Now there is a breach separating the dam into two parts asymmetricaly. The breach is assumed occurring instantaneously. In his paper, Fennema set $h_{l}=10 \mathrm{~m}, h_{r}=5 \mathrm{~m}$ and the domain consisting of a $200 \mathrm{~m} \times 200 \mathrm{~m}$ region which is subdivided into a $41 \times 41$ square grid. The breach is $75 \mathrm{~m}$ wide and centered at $75 \mathrm{~m}$. However, Alcrudo (1994) argued that the downstream water depth $5 \mathrm{~m}$ is not a severe test for the model because the flow is subcritical everywhere. Therefore, the much smaller downstream water depth should be tested for the numerical scheme. Test results after $5 \mathrm{~s}$ with three downstream water depth $5 \mathrm{~m}$ and $0 \mathrm{~m}$ are shown in the Fig. 8. The problem configuration is keep consistently with Fennema. All boundaries are solid. The time step is $0.1 \mathrm{~s}$ and the number of smoothing is 5 for each time step.

These results are very similar to others found in the literature for this problem (Anastasiou, 1997). Both time matching methods $T 1$ and $T 2$ simulated very well subcritical flows but the semi-implicit approach misses the true reality in confronting with supercritical flows while the third order Adams-Bashforth not. 

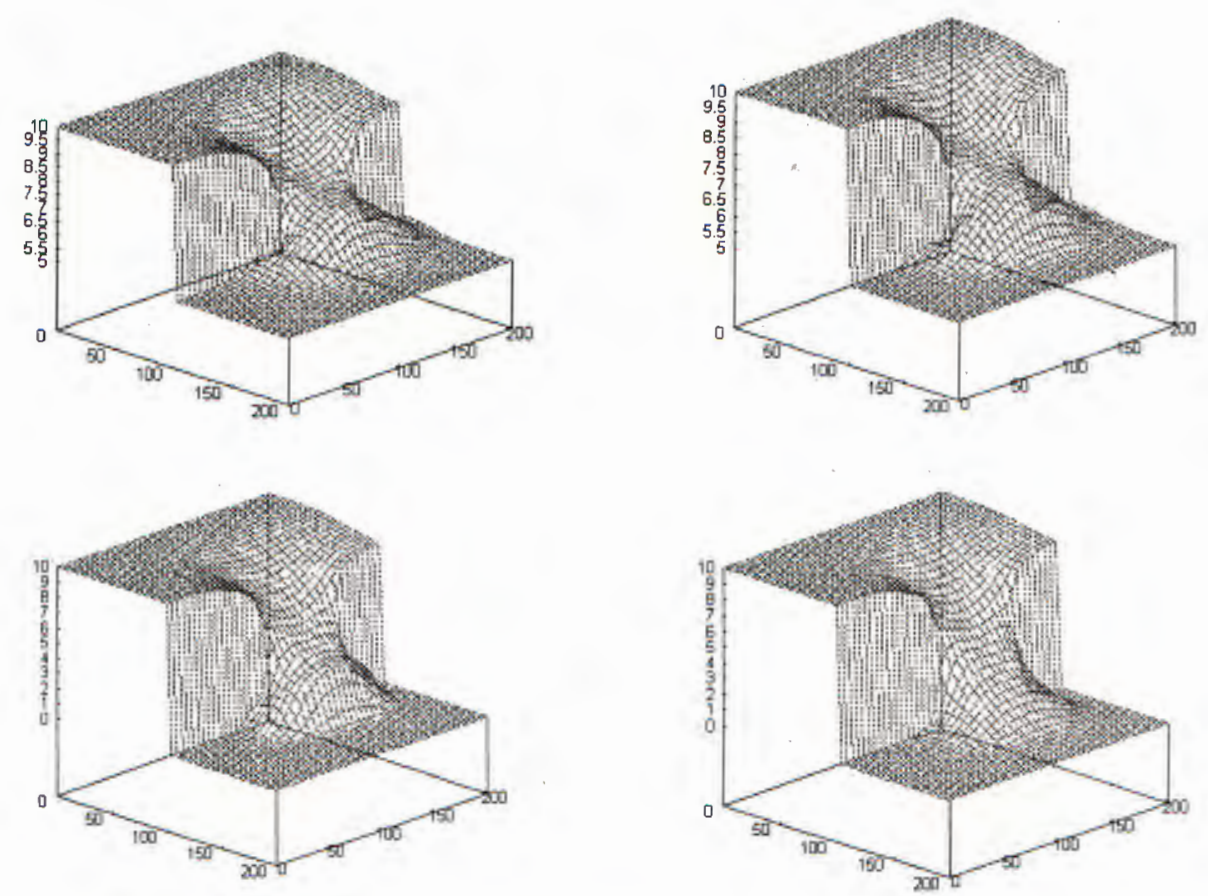

Fig. 8. The numerical results integrated after 5 s for partial dam break over a flat bed with the downstream initial water depth $5 \mathrm{~m}$ (top) and $0 \mathrm{~m}$ (bottom), which use the spatial scheme S1. The results with the semi-implicit scheme $T 1$ are in the left and the third order Adams-Bashforth scheme $T 2$ in the right.

\subsection{Reflection of a surge wave}

This test is adopted from $\mathrm{Hu}$ [4]. Friction and diffusion is ignored. The geometry is a rectangular channel $10 \mathrm{~km}$ long and $50 \mathrm{~m}$ width. One end of the channel is closed with a solid wall. The other end of the channel is an inlet boundary where a surge with water depth $d_{1}=10 \mathrm{~m}$ is imposed. Initially, water level is at rest $d_{2}=5 \mathrm{~m}$. The surge wave travels from left to right. When the wave hits the right solid wall, a reflected surge wave with increased depth of $d_{3}$ travels in the opposite direction.

The numerical results compared to the analytical results are shown in the Fig. 9. The left column shows the results after $200 \mathrm{~s}$, the right $1000 \mathrm{~s}$. The spatial step is $25 \mathrm{~m}$, the time step is $1 \mathrm{~s}$. Now the semi-implicit approach reveals large phase errors. These results are the same the results in 3.1. For discontinuous problems, the third order Adams-Bashforth should be used.

\section{CONCLUSION}

If the semi-implicit integrating scheme is considered, the directional derivative technique in spatial derivative approximation should be used. With the third order AdamsBashforth scheme, we can choose any approximation technique, which do not impact on the results significally. Between two time matching methods, the Adams-Bashforth is the most accurate. 

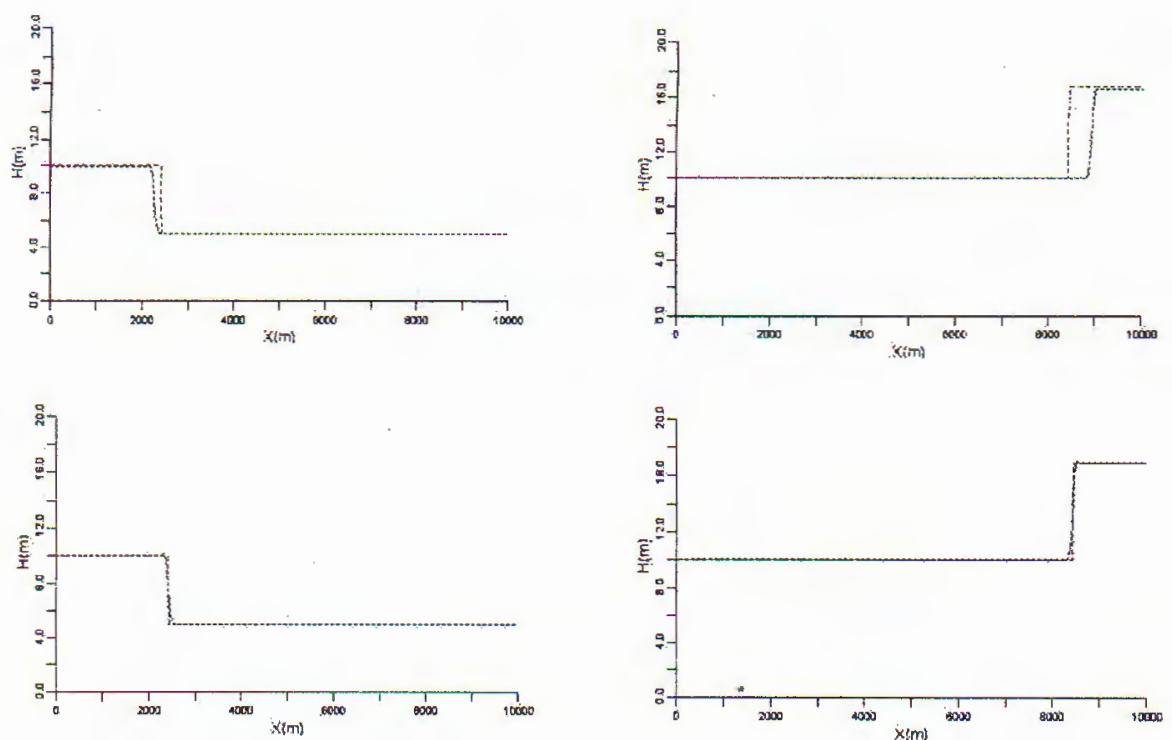

Fig. 9. The numerical solutions compared to the analytical solutions after $200 \mathrm{~s}$ (left) and 1000 s (right) for surge wave over a flat bed. The results with the schemes $S 2, T 1$ in the top and the schemes $S 1, T 4$ in the bottom.

All numerical results in testing show a good appropriation with results in literature. This leads to an impression on a good performance of the model. At least, we can assert the model quality in simulating discontinuous problems (some problems do not shown in this paper). More severe tests from continuous problems are needed to validate the model for such problems.

Spurious waves occur in the results for any time matching methods. That mean spurious waves come from the approximation techniques of spatial derivatives. And smoothing is necessary in modeling. Then we confront a problem concerning the conservation of mass. This problem is not severe in the above tests but we need examine it in future. The most difficult thing lies in choosing an appropriate number of smoothing. Large numbers of smoothing will smooth the solution drastically, while small numbers of smoothing support spurious waves and the solutions will be distorted. We can say about a sensitivity of solutions on number of smoothing.

Acknowledgement. This paper is partially supported by the basic research program numbered 301906 in ocean dynamics and environment.

\section{REFERENCES}

1. F. Alcrudo, P. G. Navarro, Computing two dimensional flood propagation with a high resolution extension of McCormack's method, Proceedings on Modelling of Flood Propagation over Initially Dry Areas, American Society of Civil Engineers, Milan, Italy, 1994; 3-17.

2. K. Anastasiou, C. T. Chan, Solution of the 2D shallow water equations using the finite volume method on unstructured triangular meshes, International Journal for Numerical Methods in Fluids 24 (1997) 1225-1245. 
3. R. J Fennema, M. H. Chaudhry, Explicit methods for 2D transient free surface flows. American Society of Civil Engineers, Journal of Hydraulic Engineering (1990) 10131034.

4. K. Hu, C. G Mingham, Causon DM. Numerical simulation of wave overtopping of coastal structures using the non-linear shallow water equations, Coastal Engineering 41 (2000) 433-465.

5. H. Lomax, T. H. Pulliam, Fundamentals of Computational Fluid Dynamics, NASA Ames Research Center 1999.

6. P. A. Madsen, O. R. Sørensen, H. A. .Schäffer, Surf zone dynamics simulated by a Boussinesq type model, Part I: Model description and cross-shore motion of regular waves, Coastal Engineering 32 (1997) 255-287.

7. K. D Nguyen, 2D shallow water models using unstructured finite volumes methods, University of Caen, France, 2002.

8. G. L. Tran, M. S Nguyen, V. C Le, Calculation of the horizontal two dimensional unsteady flows by the method of characteristics, Vietnam Journal of Mechanics $\mathbf{2 5}$ (2003) 49-64.

Received August 17, 2006

Revised December 12, 2006

\section{HAI PHƯƠNG PHÁP XẤP Xì DẠO HÀM KHÔNG GIAN TRÊN LƯ ỚI TAM GIÁC PHI CẤU TRÚC VÀ ỨNG DỤNG TRONG TÍNH TOÁN DÒNG CHẢY HAI CHIỀU}

Hai phương pháp xấp xỉ đạo hàm không gian (sử dụng định lý Green và sử dụng đạo hàm hướng) được áp dụng trong sai phân hữu hạn trên lưới tam giác phi cấu trúc. Hai phương pháp đều có độ chính xác bậc nhất. Cùng với sơ đồ tích phân thời gian bậc ba Adams-Bashforth, ba sơ đồ tích phân bán ẩn được sử dụng tích phân hệ phương trình nước nông dưới dạng không bảo toàn hoặc bảo toàn. Trong xử lý khô ướt, cách tiếp cận độ sâu ướt cực tiểu được sử dụng. Để loại bỏ các sóng nhiễu xuất hiện khi tích phân, mô hình sử dụng kỹ thuật làm trơn. Kiểm thử mô hình được thực hiện trên lưới chữ nhật đã được tam giác hóa thông qua chiến thuật sử dụng tám điểm kề. Kết quả cho thấy ba sơ đồ tích phân bán ẩn có hiệu năng như nhau. Khi sử dụng những sơ đồ này kỹ thuật đạo hàm hướng "cho lời giải tốt hơn so với kỹ thuật sử dụng định lý Green. Sơ dồ tích phân thời gian bậc ba Adams-Bashforth cho kết quả chính xác hơn cả, dặc biệt với các bài toán gián đoạn. Khác với các sơ đồ bán ẩn, sơ đồ Adams-Bashforth không cho thấy khạ́c biệt đáng kể giữa hai kỹ thuật xấp xỉ đạo hàm không gian. 